\title{
A Centralized Wavelength Resource Allocation Algorithm in SDON
}

\author{
Siyuan Liang \\ Shaanxi Key Laboratory of Information Communication Network and Security (Xi'an University of \\ Posts and Telecommunications), Xi'an 710121, China \\ telestorm@163.com
}

Keywords: SDON, Resource allocation, Resource utilization.

\begin{abstract}
Based on the research and analysis of the software defined optical networks (SDON) resource allocation mechanism, this paper proposes a new wavelength resource allocation algorithm, key link capacity-based algorithm (KLCAB). This algorithm more accurately describes the wavelength resource allocation impact, and simulation results shows KLCAB can keep lower blocking rate, which proves it improves whole network resource utilization.
\end{abstract}

\section{Introduction}

On the service-aware, the bandwidth requirements for networks will rapid growth. Different services have different requirements on resource allocation and path building in networks. The optical network is foundation of transmission networks, which needs to enlarge the capacity and to be intelligent. The controller collects services requirement then to allocate bandwidth resource in centralized method, which can improve resource utilization. The SDON introduce the SDN in optical networks based on OpenFlow extended [1-3]. SDON has an independent control plane, can support centralized resource allocation.

Based on analysis of previous algorithm this paper proposes a wavelength resource allocation KLCBA is a centralized resource allocation algorithm with whole network state. This algorithm more accurately describes the impact to of resource allocation to other paths, to improve whole network resource utilization.

\section{SDON Control Plane and Hardware Abstractions}

SDON is SDN extend to optical networks and get an independent control plane, shows in Fig 1.

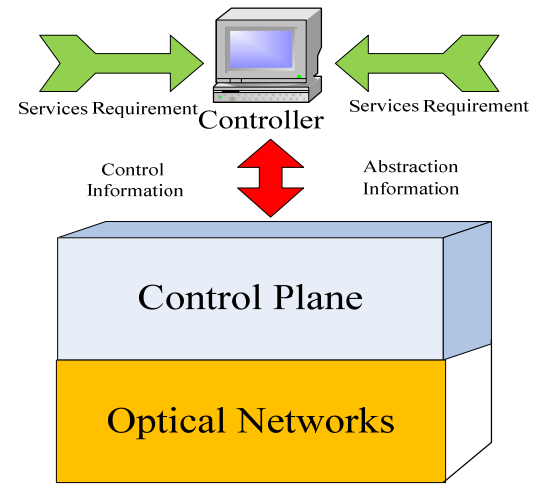

Fig 1. SDON control plane function

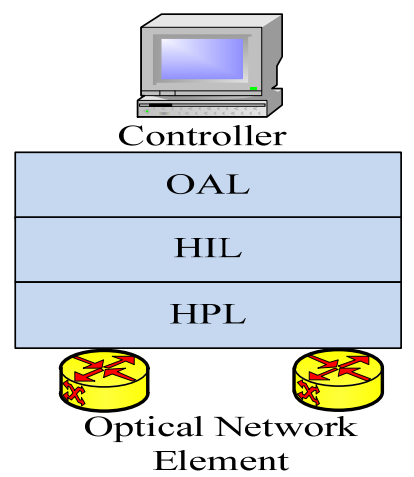

Fig 2. SDON hardware abstraction process The controller collects services requirement in application programming interface (API), and based on service-aware allocate bandwidth resource, the allocation process is constrained by services requirement and resource utilization.

The control plane completes hardware abstraction to send the optical network element physical information to controller, which can get whole networks physical resource condition [4], as Fig. 2.

The hardware abstraction shows in different layers which include hardware presentation layer (HPL), hardware interface layer (HIL), and an OpenFlow application programming interface layers 
(OAL). The HPL provides all the capabilities of the device; the HIL utilizes the raw interfaces provided by the HPL components to build useful abstractions. The SDON's hardware abstraction process can support centralized resource allocation.

\section{Key Link Capacity Based Algorithm}

\subsection{Network Model}

The KLCBA is suitable for multi-fiber network model, the definition of channel and key link can refer to relative least impact (RLI) algorithm. The path capacity is described by the number of available channels at each wavelength. $L(p)$ represents the set of links of path $\mathrm{p}, L_{c}\left(l_{i j}, \lambda_{i}\right)$ is the number of available channels for the wavelength $\lambda_{i}$ on the link $l_{i j}$, the number of available channels at any path $\mathrm{p}$ on wavelength $\lambda_{i}$ is

$$
P_{C}\left(p, \lambda_{i}\right)=\min _{l_{i j} \in L(p)} L_{c}\left(l_{i j}, \lambda_{i}\right)
$$

For the link $l_{i j} \in L(p)$, if $P_{C}\left(p, \lambda_{i}\right)=L_{c}\left(l_{i j}, \lambda_{i}\right)$, the $l_{i j}$ as the key link on path $\mathrm{p}$, because the capacity of the path is influenced by channels number on the key link. If the path has more key links, the impact probability from other wavelength resource allocation is greater. The total number of key links in path $\mathrm{p}$ on the $\lambda_{i}$ is represented by $B_{c}\left(p, \lambda_{i}\right)$, as

$$
B_{c}\left(p, \lambda_{i}\right)=\sum_{l_{i j} \in L(p) \cap L\left(p^{*}\right)} D\left(L_{c}\left(l_{i j}, \lambda_{i}\right), p_{c}\left(p, \lambda_{i}\right)\right)
$$

The function,

$$
D(A, B)=\left\{\begin{array}{rr}
1 & A=B \\
0 & A \neq B
\end{array}\right.
$$

The available wavelength set on path $p$ is

$$
A(p)=\left\{\lambda_{1}, \lambda_{2}, \cdots, \lambda_{w}\right\}
$$

\subsection{Algorithm Description and Analysis}

When the KLCBA algorithm is used to allocate the wavelength resource, it is necessary to consider the variation of available channel number on each wavelength in each path after the current wavelength allocation. KLCBA algorithm defines the affected path set $I\left(p^{*}, \lambda_{i}\right)$ to describe the influence scopes from current wavelength allocation, it denotes influenced paths set on $\lambda_{i}$ when path $p^{*}$ is assigned to $\lambda_{i} . I\left(p^{*}, \lambda_{i}\right)$ is described as:

$$
\left.\begin{array}{l}
p_{c}^{\prime}\left(p_{k^{\prime}}, \lambda_{i}\right)<p_{c}\left(p_{k^{\prime}}, \lambda_{i}\right), p_{k} \in I\left(p^{*}, \lambda_{i}\right) \\
p_{c}^{\prime}\left(p_{k^{\prime}}, \lambda_{i}\right)=p_{c}\left(p_{k^{\prime}}, \lambda_{i}\right), p_{k} \notin I\left(p^{*}, \lambda_{i}\right)
\end{array}\right\}
$$

For the above definitions: $p_{c}^{\prime}\left(p_{k}, \lambda_{i}\right)$ is the number of available channels of path $p_{k}$ when $\lambda_{i}$ is allocated to $p^{*}$, that is used to quantify the impact of current assignment process. When we calculate $p_{c}^{\prime}\left(p_{k}, \lambda_{i}\right)$ on all wavelengths, considering the path $p^{*}$ all links minus the number of channels occupied by the service, which lead to the change of available channel on the $p_{k}$ key link.

The reason of path $p_{k}$ channels can be influenced by the wavelength assignment of path $p^{*}$, that is link set $L\left(p^{*}\right)$ and the link set $L\left(p_{k}\right)$ have the sharing link, as:

$$
L\left(p_{k}\right) \cap L\left(p^{*}\right) \neq \varnothing
$$

Based on the $I\left(p^{*}, \lambda_{i}\right)$, the KLCBA algorithm defines the impacting available channel $C\left(p^{*}, \lambda_{i}\right)$, as:

$$
C\left(p^{*}, \lambda_{i}\right)=\sum_{p \in I\left(p^{*}, \lambda_{i}\right)} p_{c}^{\prime}\left(p, \lambda_{i}\right)
$$

The $C\left(p^{*}, \lambda_{i}\right)$ describes impacting of current wavelength assignment to path capacity of $I\left(p^{*}, \lambda_{i}\right)$.

The capacity factor $C_{K L C}\left(p^{*}, \lambda_{i}\right)$ is key parameters of KLCBA algorithm, which is used to determine the wavelength allocating process. 


$$
\frac{\max \lambda_{i} \in A\left(p^{*}\right) C_{K L C}\left(p^{*}, \lambda_{i}\right)=}{\sum_{p \in I\left(p^{*}, \lambda_{i}\right)} p_{c}^{\prime}\left(p_{k}, \lambda_{i}\right)}
$$

From equation (8), in the available wavelength set $A\left(p^{*}\right)$, the maximum $C_{K L C}\left(p^{*}, \lambda_{i}\right)$ value wavelength is used as the allocated wavelength. The bigger $C_{K L C}\left(p^{*}, \lambda_{i}\right)$ means that available channels are impacted less by the current wavelength allocation and the number of key links is relatively few.

\subsection{Cbr Algorithm Steps}

KLCBA is applied to the fixed routing and alternate routing. The following is the algorithm step of KLCBA in fixed routing;

Step 1 Wait for the optical path request, if any request arrives, to go to step 2;

Step 2 Analyze whether optical path request's $A(p)$ is null, if not, to go to step 3; if empty, to go to step 6;

Step 3 According to the channels number occupied by the arrival service, calculate the path set $I\left(p^{*}, \lambda_{i}\right)$ that is affected by each wavelength, go to step 4 ;

Step 4 According to $I\left(p^{*}, \lambda_{i}\right)$ calculates $C\left(p^{*}, \lambda_{i}\right)$, to step 5;

Step 5 Based on KLCBA algorithm to calculate $C_{K L C}\left(p^{*}, \lambda_{i}\right)$ of all useful wavelengths, the biggest $C_{K L C}\left(p^{*}, \lambda_{i}\right)$ wavelength is allocated to the optical path request. When the wavelength is allocated, go to step 7;

Step 6 The optical path request is rejected, and the rejected number adds 1, to go to step 1;

Step 7 The optical path request is accepted and then accepted number adds 1, to go to step 1;

When the algorithm is applied to alternate routing, the algorithm steps are the same. If you cannot allocate on the first path, jumping to the next alternative path.

\section{Simulation and Results}

The KLCBA, RLI and first fit (FF) algorithm algorithms are simulated. We suppose that the arrival rate of all service requests obeys the Poisson distribution of the mean $\beta$. The duration of the established service connection is subject to the negative exponential distribution of the mean $1 / \mu$. That is, the total load of the networks is $\beta / \mu$ erl. Take $\mu=1$, use the $5 \times 5$ grid ring network. Assume that the network does not support the queuing, if there are blocking, the service is denied.

In fixed routing, Fig. 3(a) shows KLCBA, RLI and FF algorithm blocking rate varies with network loading. In general, the blocking rate of KLCBA and RLI is significantly lower than that of FF. Comparing KLCBA with RLI, KLCBA's blocking rate is always less than RLI's blocking rate. At the beginning, the load is 25erl, RLI and KLCBA block rate have a difference of 0.0014 . Currently, due to the small load, the business has a low occupancy rate of channel resources in the network. The blocking rate is relatively low, so the RLI has a relatively small gap with KLCBA. In the end, when the load was 500erl, the RLI blocking rate and KLCBA had a difference of 0.0016, at this time, due to the large load, the channel resource occupancy rate is high, and the blocking rate is relatively high. In this condition, the main factor to impact blocking rate is the insufficient network resources, especially when the network load is large enough, KLCBA and RLI will tend to have the same blocking rate. And in the middle, when the load was 200erl, blocking rate difference is about 0.0407 between RLI and KLCBA. On moderate loading, the channel resource occupancy rate also is moderate, the algorithm itself has the most obvious effect on network blocking rate, at this stage, it is obvious that the optimization effect of KLCBA is better than RLI. 


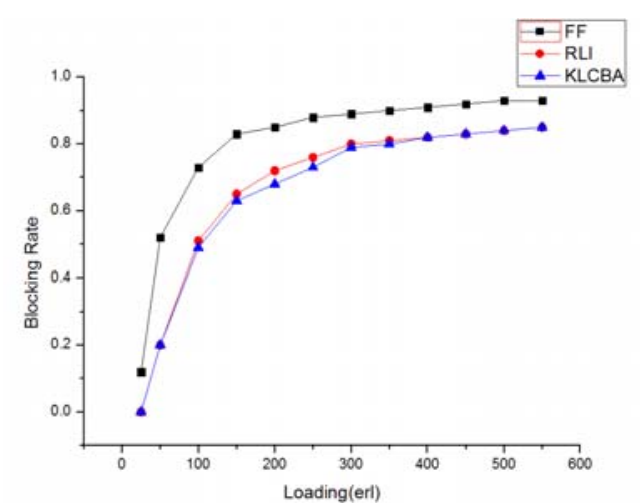

(a)



(b)

Fig 3. CBR, RLI and FF algorithm block rate comparison

Based on previous simulation, the number of available channels for each wavelength is doubled, simulation results show that the curve of KLCBA, RLI and FF algorithm blocking rate varies with network load in Fig. 3(b).

As showing in the Fig. 3(b), when the network loading is smaller, such as 25erl, 50erl, and 100erl, the maximum difference between KLCBA blocking rate and RLI is 0.0509 . When the load is greater than 200erl, the blocking rate difference between KLCBA and RLI keep in a relatively stable area of $0.0918 \sim 0.1368$. This indicates that the extreme cases are removed, such as extremely sufficient or insufficient bandwidth resources, the KLCBA has a more stable optimization effect than RLI.

\section{Summary}

Based on analysis of previous algorithm this paper proposes a wavelength resource allocation, the KLCBA is proposed in this paper. The KLCBA can be used in SDON to complete wavelength resource allocation in centralized way. It is considering overall networks resource condition, which can improve the resource utilization. The simulation results prove KLCBA have better performance than RLI.

\section{Acknowledgments}

This work was supported by Scientific Research Plan Projects of Shaanxi Education Department (No. 17JK0702) and National Natural Science Foundation of China under Grant 61371087.

\section{References}

[1]. Akhilesh Thyagaturu, Anu Mercian, Michael P. McGarry, Martin Reisslein, and Wolfgang Kellerer. Software Defined Optical Networks (SDONs): A Comprehensive Survey[J]. IEEE Communications Surveys \& Tutorials, Vol. 18 (2016) No.4, p.2738 2786.

[2]. Mingming Chen, Guochu Shou, Yihong Hu, ZhigangGuo, Guoying Zhang, Hui Ding, "Enabling Software-Defined Optical Networks Based on OpenFlow Extension"[C], Opto-Electronics and Communications Conference (OECC), ShangHai China, 2015, p. 1-3.

[3]. Yongjian Wei, Junhu Guo, Hui Li, Yuefeng Ji. Experimental demonstration of centralized control mechanism over all-optical network based on OpenFlow protocol[C], Optical Fiber Communications Conference and Exhibition (OFC), San Francisco USA, 2014, p. 1-3.

[4]. Mayur Channegowda, Reza Nejabati, and Dimitra Simeonidou. Software-Defined Optical Networks Technology and Infrastructure: Enabling Software-Defined Optical Network Operations[J]. IEEE/OSA, J. OPT. COMMUN. NETW, Vol. 5(2013) No.10, p. 274 282. 